\title{
Nonlinear Predictive Control of Shank Movement Around the Knee Joint ${ }^{\star}$
}

\author{
Amélie Chevalier* Clara M. Ionescu* Robin De Keyser* \\ * Department of Electrical energy, Systems and Automation, Ghent \\ University, Technologiepark 913, B-9052 Gent, Belgium. \\ Tel: 0032-9-264-55-80 \\ e-mail: $\{$ Amelie.Chevalier, ClaraMihaela.Ionescu, \\ Robain.DeKeyser\}@UGent.be.
}

\begin{abstract}
This paper derives an elementary mathematical model for the movement of the shank around the knee joint. The resulting equation of motion is a nonlinear differential equation. The control purpose is to regulate the angle of the shank by manipulating the torque applied to the knee joint. This model has applications in dynamical knee rigs and active orthoses. As the system is nonlinear with a rather larger range of motion, a nonlinear predictive controller is designed. The implemented control strategy is NEPSAC (Nonlinear Extended Prediction SelfAdaptive Control). The effect of the filter parameter and the prediction horizon in the NEPSAC algorithm are investigated using MATLAB/SIMULINK. The results show that for both the filter parameter and the prediction horizon an optimal value can be chosen as a trade-off between robustness and fastness of the response.
\end{abstract}

\section{INTRODUCTION}

The knee joint is the biggest and most complicated joint in the entire human body. As a result, understanding its biomechanics has been for decades the focus of research (Maquet [1984], Pitkin [2010]). In order to develop new treatments for injured knee joints or prevent future injuries, insight into the biomechanics of the knee joint is of crucial importance.

As the knee joint is the most complicated joint in the human body, it is also most exposed to injuries. There are two major reasons for knee injuries. Firstly, statistics show that knee injuries account for 15-50\% of all sports injuries (De Loës [2000]). Secondly, demographics show that there is a considerable aging in the population which coincides with a high number of knee injuries resulting from wear and tear. Two major courses of treatment for knee injuries are: total knee replacement (TKR) or active orthosis.

The first course of treatment is a TKR which will eventually be unavoidable for many patients with severe knee injuries. Consequently, the amount of TKRs performed in the European Union (EU) has increased more than $25 \%$ in the last five years to 109 TKR surgeries per 100000 population in 2012 (OECD [2012]). In the EU, a TKR surgery has an average cost of 9000 Euro per procedure (Surgery Price [2013]). An important device to evaluate the performance of TKR, develop new surgical techniques and gain insight in knee abnormalities, is a dynamical knee rig (Shaw [1973], Walker [1997], Van Haver [2013], Miller [1998], Luyckx [2009]). A dynamical knee rig is designed to mimic the natural movement of the knee joint and to impose this movement to post-mortem knee

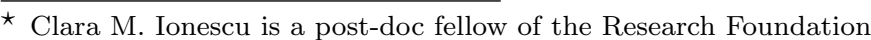
- Flanders (FWO). This research is supported by Flemish Research Foundation - Research Project FWOPR2013 005101.
}

joints or mechanical knee joints. The knee rig imposes a squat movement of the knee as occurs in daily movements such as riding a bicycle, climbing chairs, sitting down or rising from a chair (Zavatsky [1997]). However, nonlinearities, parameter uncertainties, modeling errors and external disturbances, imply a necessity for a robust closed-loop controller in order to guarantee natural movement of the knee joint.

A second course of treatment are active orthoses (Saber [2013]). Active orthoses help patients living with knee injuries regain natural movement by assisting and empowering the human movement. Orthoses include actuators that produce the joint torques needed both to compensate gravity and loading forces and to recreate natural human movements. In order to apply the correct torques to the knee joint, closed loop control is again necessary.

Current control strategies include visual control or control of the quadriceps force for the dynamical knee rig Kirsch [1998]. Active orthoses are currently controlled by PI, PD or PID controllers (Nikitczuk [2006], Pratt [2004], Costa [2006]) but also through measurements of electromyogram (EMG) signals (Fleischer [2008]).

This paper describes an elementary model of the movement of the lower part of the leg, i.e. the shank, around the knee joint. The movement of the shank around the knee joint is defined by a nonlinear equation of motion. To control the angle of the shank during the movement, the authors choose to design a nonlinear predictive controller (De Keyser [2003]).

This paper is structured as follows: the next section gives the derivation of the theoretical and mathematical model of the movement of the shank around the knee joint. Section three explains the applied nonlinear predictive controller i.e. NEPSAC. The fourth section gives simu- 
lations and results. A summary of this paper is given in a conclusion section.

\section{MODELING}

In order to design a control strategy for the shank movement around the knee joint, a 2D mathematical model is necessary to derive the nonlinear equation of motion of the system, i.e. only movement in the sagittal plane is considered. Figure 1 shows the biomechanical model of the movement of the shank when the upper leg is fixed in a horizontal position i.e. the patient is laying in a supine position (on the back).

A sketch of an active orthosis for the human leg is shown in figure 2 . This system is related to the derived mathematical model.

The biomechanical model is made up by two segments representing the upper part of the leg i.e. the femur and the lower part of the leg i.e. the shank (combination of tibia and fibula). A revolute joint connects both segments. The input of the biomechanical model is the applied torque $T$ on the knee joint. In this model the range of motion of the angle $\theta$ is limited to $90^{\circ}$ i.e. the shank can move from a horizontal position to a vertical position (for a larger range of motion, the current model needs to be extended which is the focus of future work). The value $\theta=0$ corresponds with the position in the middle of this range of motion, i.e. a position at $45^{\circ}$ from the vertical line. Therefore $\theta=45^{\circ}$

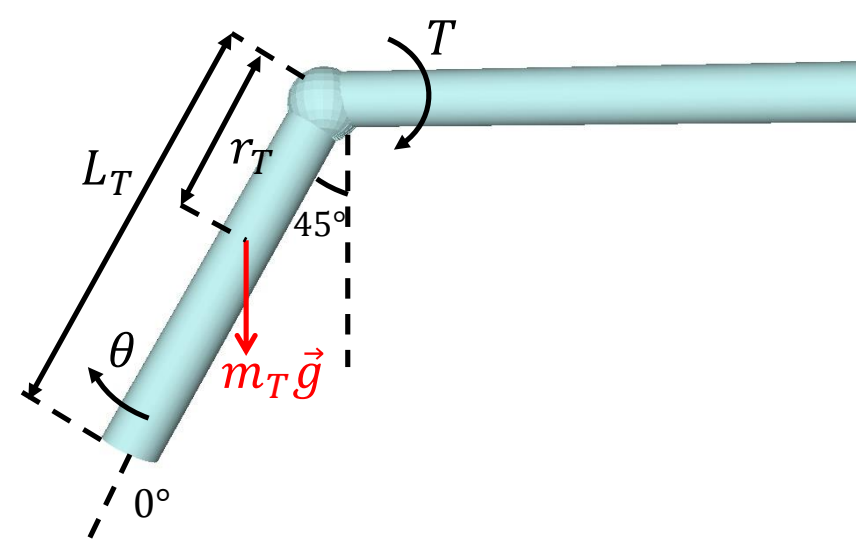

Fig. 1. Biomechanical model of the shank movement (see text for nomenclature).

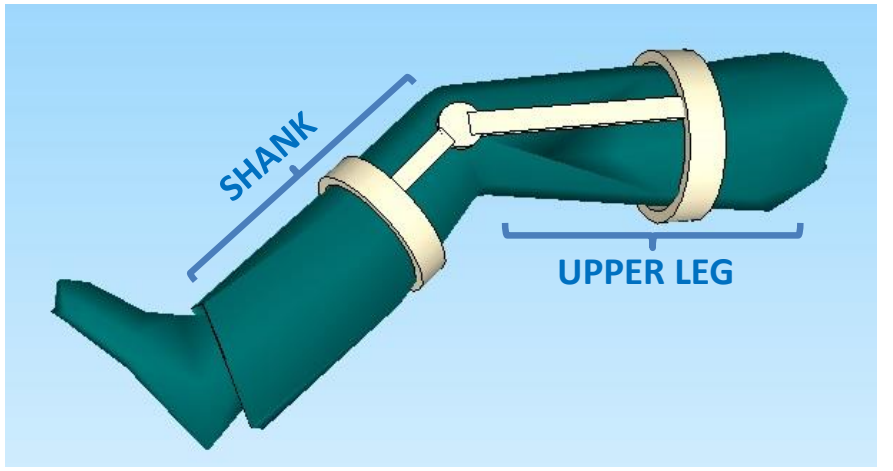

Fig. 2. Sketch of human system related to the model. corresponds to full extension of the knee joint. The angle $\theta$ is the output of the described system.

This model contains the following model parameters:

- $L_{T}$ which represents the length of the shank (0.435 $\mathrm{m})$,

- $r_{T}$ which is the distance between center of mass of the shank and the knee joint axis $(0.188 \mathrm{~m})$,

- $m_{T}$ which is the mass of the shank $(3.72 \mathrm{~kg})$,

- $g$ which is the gravitational constant $(9.81 \mathrm{~N} / \mathrm{kg})$.

Values for these model parameters (given in SI units) can be found in literature (Winter [2009]).

To derive the equation of motion for this model, we observe all of the torques around the knee joint:

- a torque caused by gravity,

- a torque caused by the inertia of the shank,

- a torque due to viscous damping,

- a torque as a result of the joint stiffness,

- the applied torque $T(t)$.

Summing all these torques and taking into account their direction we get the following equation:

$$
I_{T} \frac{d^{2} \theta(t)}{d t^{2}}+B \frac{d \theta(t)}{d t}+K \theta(t)+m_{T} g r_{T} \sin (\theta(t))=T(t)
$$

with:

- $I_{T}$ the inertia of the shank around the knee joint,

- $B$ the viscous damping coefficient of the knee joint and

- $K$ the knee joint stiffness.

The value for the inertia of the shank can be found in Winter [2009] as $0.4419 \mathrm{kgm}^{2}$. The values for the viscous damping coefficient and the joint stiffness were determined in Zhang [1998] $(K=42.2102 \mathrm{Nm} / \mathrm{rad}$ and $B=6.7453$ Nms/rad).

The obtained equation of motion for this model is a second order nonlinear equation:

$$
0.44 \frac{d^{2} \theta(t)}{d t^{2}}+6.75 \frac{d \theta(t)}{d t}+42.21 \theta(t)+6.86 \sin (\theta(t))=T(t)
$$

There are different possibilities to deal with nonlinearities in models. Linearization is a possible solution. Here the nonlinearities are approximated by linear equivalents. This is possible if the dynamics of the system stay in a narrow range around an operating point. However, for this system the range of motion is rather large i.e. $90^{\circ}$. Therefore, linearization is not the appropriate method. Another possibility to deal with nonlinearities in models is to design a nonlinear controller. In the next section, a nonlinear predictive controller will be designed for this model.

\section{NONLINEAR PREDICTIVE CONTROL}

The control purpose is to regulate the output angle $\theta$ by manipulating the torque $T$ applied to the knee joint.

The authors choose to design a nonlinear predictive controller called NEPSAC (De Keyser [2003]). NEPSAC is an acronym for 'Nonlinear Extended Prediction Self-Adaptive Control'. In this methodology, a process model is used to 


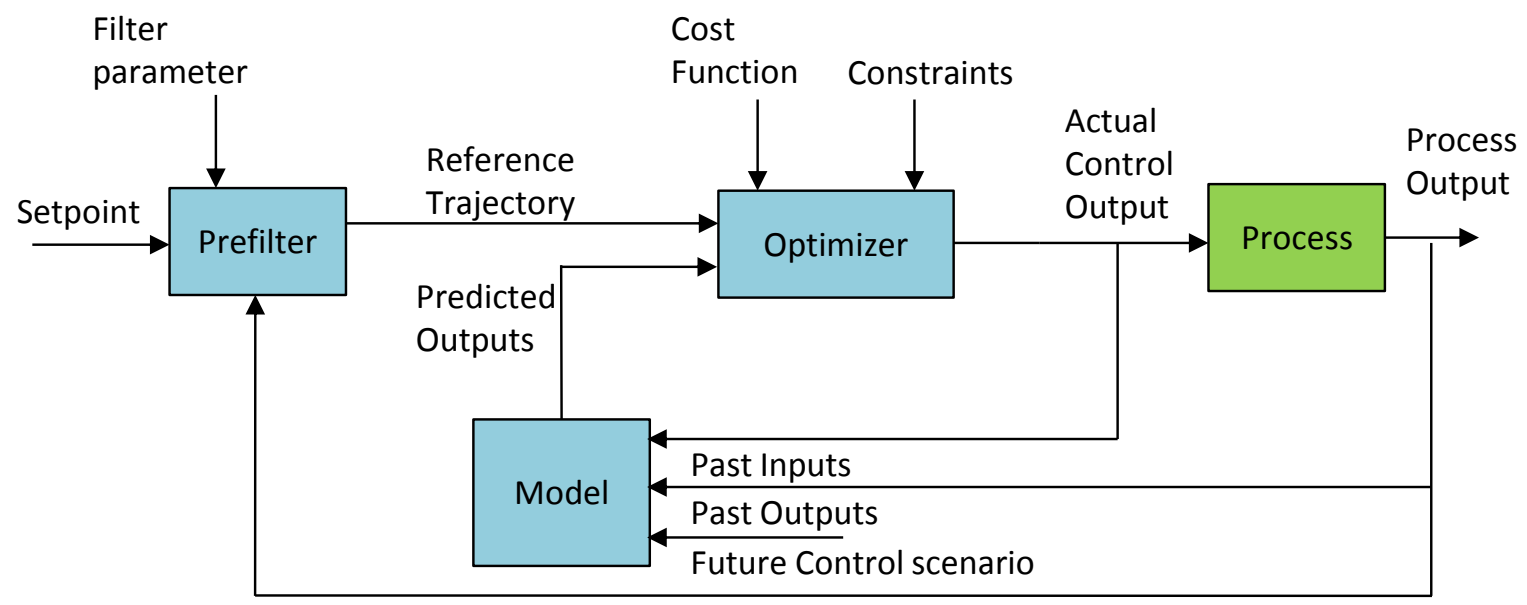

Fig. 3. Block diagram of NEPSAC strategy.

calculate a prediction of the future system output and for optimizing future control actions. The difficulty to this method is that performance of the controller depends highly on the quality of the available mathematical process model.

A block scheme of the NEPSAC algorithm is shown in figure 3. In this scheme we can see that at each current time instant the nonlinear model is used to predict the future outputs. These outputs are predicted over a certain prediction horizon $N_{2}$. We can see that the model uses information from past inputs and outputs and the future control scenario to predict the future outputs.

Over the prediction horizon $N_{2}$, a reference trajectory is defined which goes to the setpoint in a way defined by the prefilter.

An optimizer is used to calculate the control output vector. Optimization is done by minimizing a certain cost function.

\subsection{Process Model}

The process is modeled as:

$$
y(t)=x(t)+n(t)
$$

with $y(t)$ the process output, $x(t)$ the model output and $n(t)$ the process/model disturbance. The disturbance $n(t)$ can be modeled by a colored noise signal:

$$
n(t)=\frac{C\left(q^{-1}\right)}{D\left(q^{-1}\right)} e(t)
$$

with $e(t)$ a white noise signal and $C\left(q^{-1}\right)$ and $D\left(q^{-1}\right)$ are monic polynomials in the shift operator $q$.

The model output $x(t)$ represents the effect of the process input $u(t)$, i.e. the control output, on the process output $y(t)$. It is calculated using the following equation:

$$
x(t)=f[x(t-1), x(t-2), \ldots ; u(t-1), u(t-2) \ldots]
$$

where $f[$.$] is the known nonlinear process model. Using$ this equation, also the future model outputs $x(t+k \mid t)$ can be calculated for $k=1 . . N_{2}$.
In a next step the disturbance at the current time $t$ is calculated as:

$$
n(t)=y(t)-x(t)
$$

Afterwards, the future disturbances are predicted at time instant $t$ using a filtered disturbance signal $n_{f}(t)$ :

$$
n(t+k \mid t)=\frac{C\left(q^{-1}\right)}{D\left(q^{-1}\right)} n_{f}(t+k \mid t) \quad k=1 . . N_{2}
$$

with $n_{f}(t+k \mid t)=0, k=1 . . N_{2}$ and $n_{f}(t)=\frac{D\left(q^{-1}\right)}{C\left(q^{-1}\right)} n(t)$.

\subsection{The predictive controller}

The future response can be expressed as:

$$
y(t+k \mid t)=y_{\text {base }}(t+k \mid t)+y_{\text {opt }}(t+k \mid t) \quad k=1 . . N_{2}
$$

The first component $y_{\text {base }}$ is the effect of the future disturbances, the past control and the future control scenario. It is calculated as follows:

$$
y_{\text {base }}(t+k \mid t)=x(t+k \mid t)+n(t+k \mid t) \quad k=1 . . N_{2}
$$

The second component $y_{o p t}$ is the effect of optimizing the future control actions using a cost function. The future control scenario is structured using a control horizon $N_{u}$ after which the control strategy remains constant. It can be calculated as:

$\left[\begin{array}{l}y_{\text {opt }}\left(t+N_{1} \mid t\right) \\ y_{\text {opt }}\left(t+N_{1}+1 \mid t\right) \\ \cdots \\ y_{\text {opt }}\left(t+N_{2} \mid t\right)\end{array}\right]=\left[\begin{array}{l}g_{N_{1}-N_{u}+1} \\ g_{N_{1}-N_{u}+2} \\ \cdots \\ g_{N_{2}-N_{u}+1}\end{array}\right]\left[\begin{array}{l}\delta u(t \mid t) \\ \delta u(t+1 \mid t) \\ \cdots \\ \delta u\left(t-N_{u}-1 \mid t\right)\end{array}\right]$

or in matrix form:

$$
\mathbf{Y}_{\text {opt }}=\mathbf{G} \cdot \mathbf{U}
$$
$\mathbf{Y}_{\text {opt }}=\mathbf{G}$.U
with $N_{1} . . N_{2}$ the coincidence horizon and the elements of
the $\mathbf{G}$-matrix are the coefficients of the unit step response of the system. The optimal control output $\mathbf{U}^{*}$ can then be calculated as:

$$
\mathbf{U}^{*}=\left(\mathbf{G}^{\mathbf{T}} \mathbf{G}\right)^{-1} \mathbf{G}^{\mathbf{T}}\left(\mathbf{R}-\mathbf{Y}_{\text {base }}\right)
$$

with $\mathbf{R}$ and $\mathbf{Y}_{\text {base }}$ the matrix notations of respectively the reference trajectory and the signals $y_{\text {base }}(t+k \mid t)$. $\mathbf{U}^{*}$ is the value that minimizes the following cost function: 


$$
\sum_{k=N_{1}}^{N_{2}}[r(t+k \mid t)-y(t+k \mid t)]^{2}
$$

where $r(. \mid$.) represents the reference trajectory.

In the NEPSAC algorithm an iteration step is necessary to find the absolute optimal control action $\mathbf{U}^{*}$ that minimizes the optimal future response $y_{\text {opt }}$ (value close to zero). This means that the G-matrix has to be calculated for every iteration (unlike EPSAC where the $\mathbf{G}$-matrix is calculated only once).

\section{SIMULATIONS AND RESULTS}

We have designed the NEPSAC controller for the system of the knee and simulated the response in MATLAB/SIMULINK. The SIMULINK scheme for the nonlinear model is shown in figure 4 .

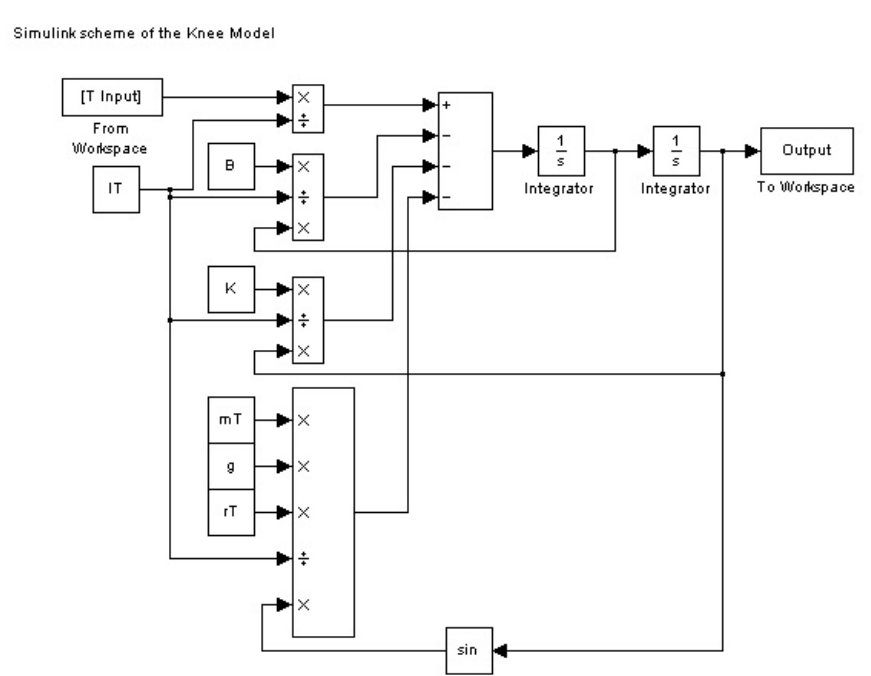

Fig. 4. SIMULINK scheme of the nonlinear model.

For implementation, the parameters of the controller are:

- the prediction horizon $N_{2}$,

- $N_{1}$ is taken to be 1 as the system does not have a time delay,

- the control horizon $N_{u}$ which is taken to be 1 (default value),

- filter parameter $\alpha$.

In the simulations, we looked at the effect of the prediction horizon and the filter parameter $\alpha$.

The setpoint signal for all simulations is the block signal shown in figure 5.

\subsection{Effect of filter parameter}

This filter parameter $\alpha$ is used in the prefilter between the setpoint $w(t+k \mid t)$ and the reference trajectory $r(t+k \mid t)$ : $r(t+k \mid t)=\alpha r(t+k-1 \mid t)+(1-\alpha) w(t+k \mid t), \quad k=1 . . N_{2}$

with initialization $r(t \mid t)=y(t)$. To investigate the effect of $\alpha$, we fixed the prediction horizon $N_{2}$ to 5 and changed the values of $\alpha$ between 0 and 1. A magnification of the

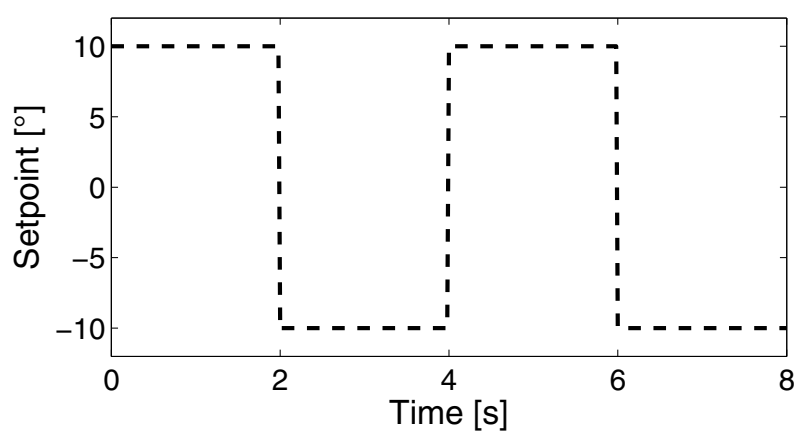

Fig. 5. Setpoint signal.
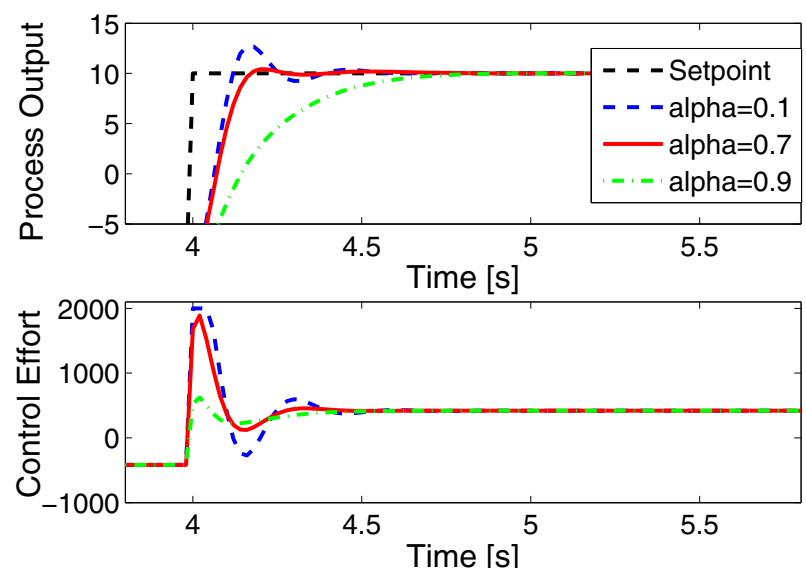

Fig. 6. The effect of the filter parameter $\alpha$ (magnification).

results is shown in figure 6 . The complete figure is shown in figure 7.

When $\alpha$ is taken to be zero the reference trajectory will be a pure step which will result in a high peak in the control output. Therefore, we can see that the control effort has high peaks for low values of $\alpha$. However, by changing the value of $\alpha$ between 0 and 1, the reference trajectory will go smoother to the desired set point and result in a normal control output. But higher values of $\alpha$ result in a slower response as can be seen in figure 7 . Therefore a trade-off between control effort and fast response is necessary. We desire an output response which is as fast as possible with a reasonable control effort. Therefore, for next simulations, the value of $\alpha$ is set to 0.7 .

\subsection{Effect of prediction horizon}

The prediction horizon $N_{2}$ is related to the system's time constant and is usually taken between $N_{1}+1$ and $N_{1}+10$. We varied therefore the value of $N_{2}$ between 2 and 11 . The result is shown in figure 8 . A magnification of figure 8 is shown in figure 9.

Figure 9 shows that for lower values of $N_{2}$ the output reaches the setpoint faster, while the response for higher values of $N_{2}$ is longer. Notice in the control effort, the sharper peaks when $N_{2}$ has a lower value. Therefore, we need a trade-off between a fast response and a normal control effort. We desire an output response which is as fast as possible with a reasonable control effort. We conclude 

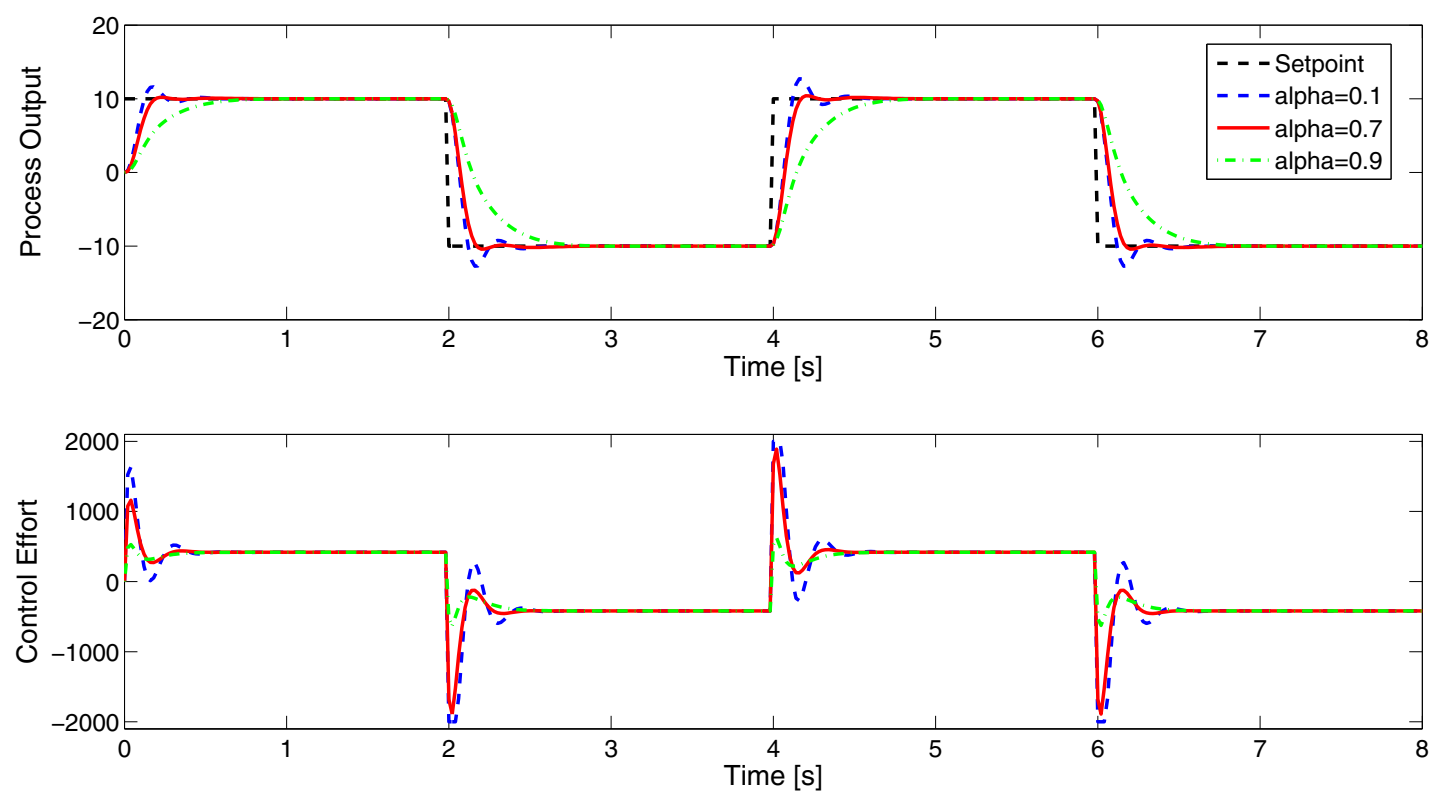

Fig. 7. The effect of the filter parameter $\alpha$.
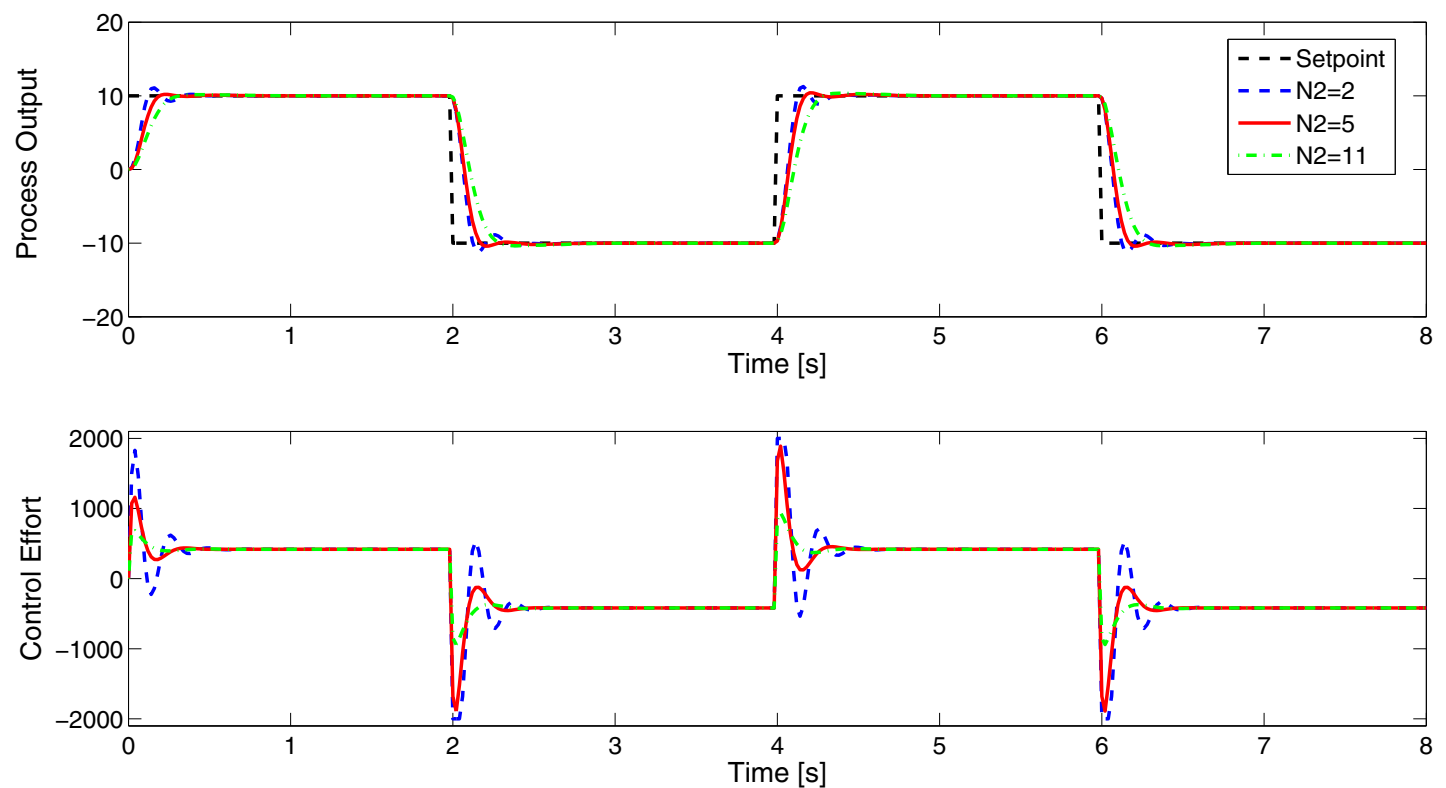

Fig. 8. The effect of the prediction horizon $N_{2}$.

that a value of $N_{2}=5$ will result in a controller that is still fast enough and has a high robustness.

Notice in figures 7 and 8 that different values of $\alpha$ and $N_{2}$ will result in responses ranging from underdamped to overdamped systems. As an overshoot is highly undesirable in the controller knee joint, the underdamped systems have to be avoided. By taking into account the physical limitations of the knee joint when selecting a reference signal, this highly undesirable effect can be avoided.

\section{CONCLUSION}

In this research a nonlinear model for the movement of the shank around the knee joint is derived. The control purpose is to regulate the output angle $\theta$ by manipulating the torque $T$ applied to the knee joint. In order to deal with the nonlinearity in the model a nonlinear predictive controller is applied to the system as the range of motion is too large to apply linearization.

The nonlinear predictive control strategy applied in this paper is NEPSAC (Nonlinear Extended Prediction SelfAdaptive Control). To design the controller, the filter pa- 
rameter and the prediction horizon need to be determined. The effect of both parameters on the results are discussed. It can be concluded that for both the filter parameter and the prediction horizon an optimal value can be found as trade-off between normal control effort without high peaks and fastness of the response.
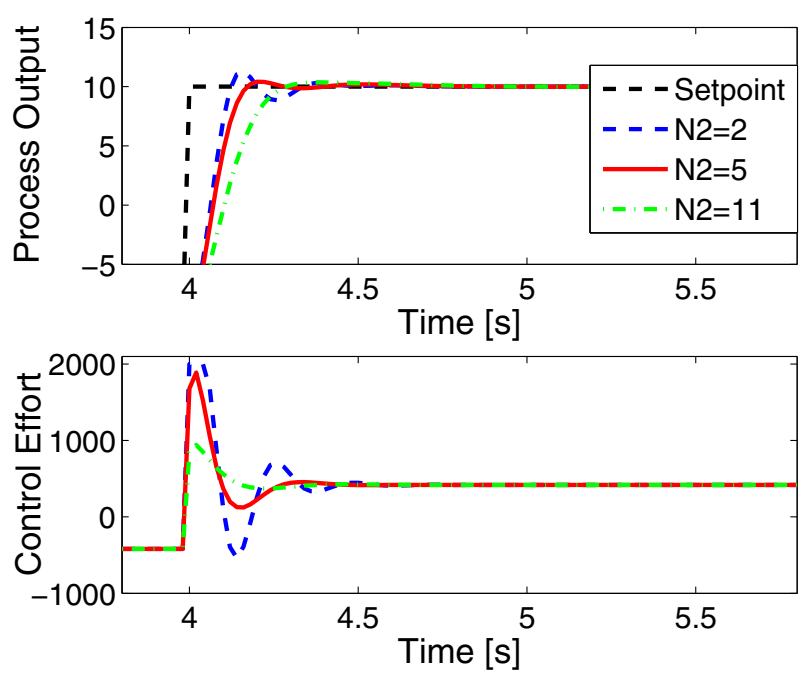

Fig. 9. The effect of the prediction horizon $N_{2}$ (magnification).

\section{ACKNOWLEDGEMENTS}

Clara M. Ionescu is a post-doc fellow of the Research Foundation - Flanders (FWO). This research is supported by Flemish Research Foundation - Research Project FWOPR2013 005101.

\section{REFERENCES}

Costa, N., Bezdicek, M. and Brown, M. (2006). Joint motion control of a powered lower limb orthosis for rehabilitation. Int. J. Autom. Comput., 3(3), 271-281.

De Loës, M., Dahlstedt, L.J. and Thomée, R. (2000). A 7year study on risks and costs of knee injuries in male and female youth participants in 12 sports. Scandinavian Journal of Medicine ES Science in Sports, 10(2), 90-97.

De Keyser, R. (2003). A 'Gent'le approach to predictive control. UNESCO Encyclopedia of Life Support Systems (EoLSS), article 6.43.16.1 p. 30.

Fleischer, C. and Hommel, G. (2008). A humanexoskeleton interface utilizing electromyography. IEEE Trans. Robot., 24(4), 872-882.

Kirsch, L. , Wirth, C.J., Kohn, D. and Glowik, A. (1998). A dynamic knee simulator with feedback control. Journal of Biomechanics, 31, 143.

Luyckx, T. , Didden, K., Vandenneucker, H., Labey, L., Innocenti, B. and Bellemans, J. (2009). Is there a biomechanical explanation for anterior knee pain in patients with patella alta? Influence of patellar height on patellofemoral contact force, contact area and contact pressure,. Journal of bone and joint surgery-British Volume, 91B(3), 344-350.

Maquet, P.G.J. (1984). Biomechanics of the knee with application to the pathogenesis and the surgical treatment of osteoarthritis. Springer-Verlag, Berlin Heidelberg, 2nd edition.

Miller, R.K., Goodfellow, J.W., Murray, D.W. and O'Connor, J.J. (1998). In vitro measurement of patellofemoral force after three types of knee replacement. Journal of bone and joint surgery- British Volume, 80B(5), 900-906.

Nikitczuk, J., Das, A. and Vyas, H. (2006). Control of electro-rheological fluid based resistive torque elements for use in active rehabilitation devices. Int. J. Robot. Autom., 16(2), 393-399.

OECD (The organisation for economic co-operation and development). Health at a Glance: Europe 2012. http://www.oecd-ilibrary.org. Accessed October 2013.

Pitkin, M.R. (2010). Biomechanics of lower limb prosthetics. Springer-Verlag, Berlin Heidelberg.

Pratt, J. E., Krupp, B. T. and Morse, C. J. (2004). The roboKnee: An exoskeleton for enhancing strength and endurance during walking. Int. J. Robot. Autom., 3, 2430-2435.

Saber, M., Samer, M. and Yacine, A. (2013). Towards movement restoration of knee joint using robust control of powered orthosis. IEEE Transactions on Control Systems Technology, DOI 10.1109/TCST.2012.2228194.

Shaw, J.A. and Murray, D.G. (1973). Knee Joint Simulator, Clinical Orthopaedics and Related Research, 94, $15-23$.

Surgery Price. Knee Replacement Price. http://www.surgeryprice.co.uk. Accessed October 2013.

Van Haver, A., De Roo, K., Claessens, T., De Beule, M., Verdonk, P. and De Baets, P. (2013). Pilot validation study on a quasi-static weight-bearing knee rig. Proceedings of the institute of mechanical engineers part $H$ journal of engineering in Medicine, 227(H3), 229-233.

Walker, P.S., Blunn, G.W., Broome, D.R., Perry, J., Watkins, A., Sathasivam, S., Dewar, M.E. and Paul, J.P. (1997). A knee simulating machine for performance evaluation of total knee replacements. Journal of Biomechanics, 30(1),83-89.

Winter, D.A. (2009). Biomechanics and control of human movement. John Wiley \& Sons, Inc., Hoboken, New Jersey, 4th edition, 82-95.

Zhang, L-Q., Nuber, G., Butler, J., Bowen, M. and Rymer, W.Z. (1998). In vivo human knee joint dynamic properties as functions of muscle contraction and joint position. Journal of Biomechanics, 31, 71-76.

Zavatsky, A.B. (1997). A Kinematic-freedom analysis of a flexed-knee-stance testing rig. Journal of Biomechanics, $30(3), 277-280$. 